\title{
Front Matter: Volume 8778
}

, "Front Matter: Volume 8778," Proc. SPIE 8778, Advances in X-ray FreeElectron Lasers II: Instrumentation, 877801 (8 May 2013); doi:

$10.1117 / 12.2030597$

SPIE. Event: SPIE Optics + Optoelectronics, 2013, Prague, Czech Republic 


\title{
PROCEEDINGS OF SPIE
}

\section{Advances in X-ray Free-Electron Lasers II: Instrumentation}

\author{
Thomas Tschentscher \\ Kai Tiedtke \\ Editors
}

\section{7-18 April 2013}

Prague, Czech Republic

Sponsored by

SPIE

Cooperating Organisations

HiPER Project (United Kingdom)

ELI Beamlines (Czech Republic)

International Commission For Optics

Published by

SPIE 
The papers included in this volume were part of the technical conference cited on the cover and title page. Papers were selected and subject to review by the editors and conference program committee. Some conference presentations may not be available for publication. The papers published in these proceedings reflect the work and thoughts of the authors and are published herein as submitted. The publisher is not responsible for the validity of the information or for any outcomes resulting from reliance thereon.

Please use the following format to cite material from this book:

Author(s), "Title of Paper," in Advances in X-ray Free-Electron Lasers II: Instrumentation, edited by Thomas Tschentscher, Kai Tiedtke, Proceedings of SPIE Vol. 8778 (SPIE, Bellingham, WA, 2013) Article CID Number.

ISSN: 0277-786X

ISBN: 9780819495808

Published by

SPIE

P.O. Box 10, Bellingham, Washington 98227-0010 USA

Telephone +1 3606763290 (Pacific Time) · Fax +1 3606471445

SPIE.org

Copyright (C) 2013, Society of Photo-Optical Instrumentation Engineers.

Copying of material in this book for internal or personal use, or for the internal or personal use of specific clients, beyond the fair use provisions granted by the U.S. Copyright Law is authorized by SPIE subject to payment of copying fees. The Transactional Reporting Service base fee for this volume is $\$ 18.00$ per article (or portion thereof), which should be paid directly to the Copyright Clearance Center (CCC), 222 Rosewood Drive, Danvers, MA 01923. Payment may also be made electronically through CCC Online at copyright.com. Other copying for republication, resale, advertising or promotion, or any form of systematic or multiple reproduction of any material in this book is prohibited except with permission in writing from the publisher. The CCC fee code is 0277-786X/13/\$18.00.

Printed in the United States of America.

Publication of record for individual papers is online in the SPIE Digital Library.

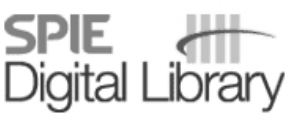

SPIEDigitallibrary.org

Paper Numbering: Proceedings of SPIE follow an e-First publication model, with papers published first online and then in print and on CD-ROM. Papers are published as they are submitted and meet publication criteria. A unique, consistent, permanent citation identifier (CID) number is assigned to each article at the time of the first publication. Utilization of CIDs allows articles to be fully citable as soon as they are published online, and connects the same identifier to all online, print, and electronic versions of the publication. SPIE uses a six-digit CID article numbering system in which:

- The first four digits correspond to the SPIE volume number.

- The last two digits indicate publication order within the volume using a Base 36 numbering

system employing both numerals and letters. These two-number sets start with 00, 01, 02, 03, 04, $05,06,07,08,09,0 A, 0 B \ldots 0 Z$, followed by 10-1Z, 20-2Z, etc.

The CID Number appears on each page of the manuscript. The complete citation is used on the first page, and an abbreviated version on subsequent pages. Numbers in the index correspond to the last two digits of the six-digit CID Number. 


\section{Contents}

vii Conference Committee

SESSION 1 STATUS OF OPERATIONAL AND PLANNED FEL FACILITIES AND SOURCE DEVELOPMENTS

$877803 \quad$ Harmonic lasing in X-ray FELs (Invited Paper) [8778-2]

E. A. Schneidmiller, M. V. Yurkov, Deutsches Elektronen-Synchrotron (Germany)

\section{SESSION 2 SCIENTIFIC APPLICATIONS AND THEIR INSTRUMENTATION REQUIREMENTS}

877807 Four wave mixing using coherent FEL radiation (Invited Paper) [8778-6]

F. Bencivenga, L. Raimondi, Sincrotrone Trieste S.C.p.A (Italy); C. Svetina, Sincrotrone Trieste S.C.p.A (Italy) and Univ. degli Studi di Trieste (Italy); C. Maschiovecchio, Sincrotrone Trieste

S.C.p.A (Italy)

877808 The impact of pulse duration on multiphoton ionization in the soft X-ray regime [8778-7] M. Richter, Physikalisch-Technische Bundesanstalt (Germany); A. A. Sorokin, K. Tiedtke, Deutsches Elektronen-Synchrotron (Germany)

\section{SESSION 3 OPTICS, BEAM TRANSPORT PERFORMANCE, SPATIAL AND COHERENCE PROPERTIES}

$8778 \mathrm{OB}$ Recent development of thin diamond crystals for X-ray FEL beam-sharing [8778-10] Y. Feng, R. Alonso-Mori, SLAC National Accelerator Lab. (United States); V. Blank, Technological Institute for Superhard and Novel Carbon Materials (Russian Federation); S. Boutet, M. Chollet, SLAC National Accelerator Lab. (United States); T. B. van Driel, SLAC National Accelerator Lab. (United States) and Technical Univ. of Denmark (Denmark); D. M. Fritz, J. M. Glownia, J. B. Hastings, H. Lemke, M. Messerchmidt, P. A. Montanez, A. Robert, J. Robinson, SLAC National Accelerator Lab. (United States); L. Samoylova, European XFEL GmbH (Germany); Y. Shvyd'ko, Argonne National Lab. (United States); M. Sikorski, SLAC National Accelerator Lab. (United States); H. Sinn, European XFEL GmbH (Germany); S. Song, V. N. Srinivasan, SLAC National Accelerator Lab. (United States); S. Stoupin, Argonne National Lab. (United States); S. Terentiev, Technological Institute for Superhard and Novel Carbon Materials (Russian Federation); G. Williams, D. Zhu, SLAC National Accelerator Lab. (United States)

8778 0C Development of active gratings for the spectral selection of ultrafast pulses [8778-11] F. Frassetto, S. Bonora, CNR, Institute of Photonics and Nanotechnologies, Politecnico di Milano (Italy); G. Brusatin, G. Della Giustina, Univ. degli Studi di Padova (Italy); S. Stagira, C. Vozzi, CNR, Institute of Photonics and Nanotechnologies, Politecnico di Milano (Italy); E. Zanchetta, Univ. degli Studi di Padova (Italy); L. Poletto, CNR, Institute of Photonics and Nanotechnologies, Politecnico di Milano (Italy) 
$87780 \mathrm{G}$ Full characterization of a focused wave field with sub $100 \mathrm{~nm}$ resolution [8778-15] R. Hoppe, V. Meier, J. Patommel, F. Seiboth, Technische Univ. Dresden (Germany); H. J. Lee, B. Nagler, E. C. Galtier, B. Arnold, U. Zastrau, J. Hastings, SLAC National Accelerator Lab. (United States); D. Nilsson, F. Uhlén, U. Voigt, H. M. Hertz, Royal Institute of Technology (Sweden); C. G. Schroer, Technische Univ. Dresden (Germany); A. Schropp, SLAC National Accelerator Lab. (United States)

$8778 \mathrm{OH}$ Measurement of wavefront and Wigner distribution function for optics alignment and full beam characterization of FELs [8778-16]

T. Mey, B. Schäfer, K. Mann, Laser-Lab. Göttingen e.V. (Germany); B. Keitel, S. Kreis,

M. Kuhlmann, E. Plönjes, K. Tiedtke, Deutsches Elektronen-Synchrotron (Germany)

\section{SESSION 4 DIAGNOSTICS OF FEL RADIATION I: ELECTRON BEAM DIAGNOSTICS}

8778 0J LCLS accelerator operation and measurement of electron beam parameters relevant for the X-ray beam (Invited Paper) [8778-18]

H. Loos, SLAC National Accelerator Lab. (United States)

$87780 \mathrm{M}$ Longitudinal bunch profile diagnostics with coherent radiation at FLASH [8777-21]

E. Hass, Univ. Hamburg (Germany); C. Gerth, B. Schmidt, Deutsches Elektronen-Synchrotron (Germany) S. Wesch, Helmholtz-Zentrum Berlin für Materialien und Energie GmbH

(Germany); M. Yan, Deutsches Elektronen-Synchrotron (Germany)

SESSION 5 DIAGNOSTICS OF FEL RADIATION II: X-RAY BEAM DIAGNOSTICS IN THE TIME DOMAIN

$87780 Q \quad$ Ultrafast laser synchronization at the FERMI@Elettra FEL [8778-25]

P. Sigalotti, P. Cinquegrana, A. Demidovich, R. Ivanov, I. Nikolov, G. Kurdi, M. B. Danailov, Sincrotrone Trieste S.C.p.A (Italy)

$8778 \mathrm{OR}$ Femtosecond-precision synchronization of the pump-probe optical laser for user experiments at FLASH [8778-26]

S. Schulz, M. K. Czwalinna, M. Felber, Deutsches Elektronen-Synchrotron (Germany);

P. Prędki, Technical Univ. of Łódz (Poland); S. Schefer, Univ. Hamburg (Germany);

H. Schlarb, U. Wegner, Deutsches Elektronen-Synchrotron (Germany)

\section{SESSION 6 FEL INSTRUMENTATION AND SAMPLE RELATED ISSUES}

8778 OS Femtosecond optical/hard X-ray timing diagnostics at an FEL: implementation and performance (Invited Paper) [8778-27]

H. T. Lemke, M. Weaver, M. Chollet, J. Robinson, J. M. Glownia, D. Zhu, SLAC National Accelerator Lab. (United States); M. R. Bionta, CNRS, Univ. de Toulouse (United States); M. Cammarata, Institut de Physique de Rennes (France); M. Harmand, Deutsches Elektronen-Synchrotron (Germany); R. N. Coffee, D. M. Fritz, SLAC National Accelerator Lab. (United States)

8778 OT Sample refreshment schemes for high repetition rate FEL experiments (Invited Paper) [8778-28]

J. Schulz, S. Bari, J. Buck, C. Uetrecht, European XFEL GmbH (Germany) 
8778 OU Status of detector development for the European XFEL [8778-29]

J. Sztuk-Dambietz, S. Hauf, A. Koch, M. Kuster, M. Turcato, European XFEL GmbH (Germany)

8778 OV The AGIPD System for the European XFEL [8778-30]

L. Bianco, J. Becker, Deutsches Elektronen-Synchrotron (Germany); R. D. Dinapoli, Paul

Scherrer Institut (Switzerland); E. Fretwurst, Univ. Hamburg (Germany); P. Goettlicher,

Deutsches Elektronen-Synchrotron (Germany); H. Graafsma, Deutsches Elektronen-

Synchrotron (Germany) and Mid Sweden Univ. (Sweden); D. Greiffenberg, Paul Scherrer Institut (Switzerland); M. Gronewald, Univ. Bonn (Germany); B. Henrich, Paul Scherrer Institut (Switzerland); H. Hirsemann, S. Jack, Deutsches Elektronen-Synchrotron (Germany);

R. Klanner, Univ. Hamburg (Germany); A. Klyuev, Deutsches Elektronen-Synchrotron (Germany); H. Krueger, Univ. Bonn (Germany); A. Marras, Deutsches ElektronenSynchrotron (Germany); A. Mozzanica, Paul Scherrer Institut (Switzerland); S. Rah, Deutsches Elektronen-Synchrotron (Germany); B. Schmitt, X. Shi, Paul Scherrer Institut (Switzerland); U. Trunk, Deutsches Elektronen-Synchrotron (Germany); J. Schwandt, J. Zhang, Univ. Hamburg (Germany)

8778 oW Instrument for single-shot X-Ray emission-spectroscopy experiments [8778-31]

L. Poletto, F. Frassetto, P. Miotti, CNR, Institute of Photonics and Nanotechnologies, Politecnico di Milano (Italy); M. Coreno, CNR, Institute of Inorganic Methodologies and Plasmas (Italy); A. Di Cicco, Univ. degli Studi di Camerino (Italy); S. Stagira, CNR, Institute of Photonics and Nanotechnologies, Politecnico di Milano (Italy)

\section{SESSION PS POSTER SESSION}

877810 Beam characterization of FLASH from beam profile measurement by intensity transport equation and reconstruction of the Wigner distribution function [8778-35]

B. Schäfer, T. Mey, K. Mann, Laser-Lab. Göttingen e.V. (Germany); B. Keitel, S. Kreis, M. Kuhlmann, E. Plönjes, K. Tiedtke, Deutsches Elektronen-Synchrotron (Germany)

877811 Tunable IR/THz source for pump probe experiments at the European XFEL [8778-36] E. A. Schneidmiller, M. V. Yurkov, M. Krasilnikov, F. Stephan, Deutsches ElektronenSynchrotron (Germany)

877813 Micro-focusing of soft X-ray pulses by grazing-incidence toroidal mirrors [8778-38] L. Poletto, F. Frassetto, S. Anumula, F. Calegari, A. Trabattoni, M. Nisoli, CNR, Institute of Photonics and Nanotechnologies, Politecnico di Milano (Italy)

877814 Hartmann wavefront measurements at FLASH [8778-39]

B. Keitel, Deutsches Elektronen-Synchrotron (Germany); B. Flöter, Laser-Lab. Göttingen e.V. (Germany); S. Kreis, M. Kuhlmann, Deutsches Elektronen-Synchrotron (Germany); K. Mann, T. Mey, E. Plönjes, B. Schäfer, Laser-Lab. Göttingen e.V. (Germany); K. Tiedtke, Deutsches Elektronen-Synchrotron (Germany)

877816 Parametric beam instability of the electron bunch in a crystal [8778-41] A. Leonov, A. Benediktovitch, Belarusian State Univ. (Belarus); D. Ksenzov, Univ. Siegen (Germany); I. Feranchuk, Belarusian State Univ. (Belarus); U. Pietsch, Univ. Siegen (Germany) 
8778 1A FLASH2 photon diagnostics and beamline concepts [8778-45]

M. Kuhlmann, E. Plönjes, Deutsches Elektronen-Synchrotron (Germany)

8778 1C A new compact design of a three-dimensional Ionization Profile Monitor (IPM) [8778-47]

H. Breede, H.-J. Grabosch, M. Sachwitz, Deutsches Elektronen-Synchrotron (Germany)

8778 1D An electron beam detector for the FLASH II beam dump [8778-48]

J. Good, G. Kube, N. Leuschner, F. Perlick, M. Sachwitz, M. Schmitz, K. Wittenburg,

T. Wohlenberg, Deutsches Elektronen-Synchrotron (Germany)

$87781 G \quad$ A split- and delay-unit for the European XFEL [8778-51]

S. Roling, Westfälische Wilhelms-Univ. Münster (Germany); S. Braun, P. Gawlitza, Fraunhofer IWS Dresden (Germany); L. Samoylova, European XFEL GmbH (Germany); B. Siemer,

Westfälische Wilhelms-Univ. Münster (Germany); H. Sinn, European XFEL GmbH (Germany);

F. Siewert, Helmholtz-Zentrum Berlin für Materialien und Energie GmbH (Germany);

F. Wahlert, M. Wöstmann, H. Zacharias, Westfälische Wilhelms-Univ. Münster (Germany)

Author Index 


\title{
Conference Committee
}

\author{
Symposium Chairs
}

J in Homola, Institute of Photonics and Electronic s of the ASC R, v.v.i

(Czech Republic)

Chris Edwards, Central Laser Fac ility, Science and Technology

Facilities Council (United Kingdom)

Mike Dunne, Lawrence Livermore National Laboratory (United States)

Ivo Rendina, CNR, Instituto per la Mic roelettronica e Mic rosistemi

(Italy)

Honora ry Symposium Chair

Miroslav Miller, Institute of Photonic s and Elec tronic s of the ASCR, v.v.i

(Czech Republic)

Conference Chairs

Thomas Tschentscher, European XFEL G mbH (Gemany)

Kai Tiedtke, Deutsc hes Elektronen-Synchrotron (Gemany)

Conference Programme Committee

Roberto Cimino, Istituto Nazionale di Fisica Nucleare (Italy)

Christopher Gerth, Deutsc hes Elektro nen-Synchrotron (Germany)

Kaus Mann, La ser-La bo ra torium Göttingen e.V. (Gemany)

Aymeric Robert, SLAC National Accelerator Laboratory

(United States)

Volker Sc hlott, Paul Scherrer Institut (Switzerland)

Makina Yabashi, RIKEN (J apan)

Marco Zangrando, Sincrotrone Trieste S.C.p.A. (Italy)

Philippe Zeitoun, Ecole Nationa le Supérieure de Techniques

Avancées (France)

Session Chairs

1 Status of Operational and Planned FEL Facilities and Source Developments

Thomas Tsc hentsc her, Euro pean XFEL G mbH (Germany)

2 Sc ientific Applicationsand Their Instrumentation Requirements

Aymeric Robert, SLAC National Accelerator Laboratory

(United Sta tes) 
3 Optics, Beam Transport Performance, Spatial and Coherence Properties

Kaus Mann, La ser-La bo ra torium Göttingen e.V. (Germany)

4 Diagnostic s of FEL Radiation I: Elec tron Beam Dia gnostics

Volker Sc hlott, Paul Scherrer Institut (Switzerland)

5 Diagnostic s of FEL Ra diation II: X-ray Beam Diagnostics in the Time Domain

Christopher Gerth, Deutsc hes Elektronen-Synchrotron (Germany)

6 FEL Instrumentation and Sample Related Issues

Marco Zangrando, Sincrotrone Trieste S.C.p.A. (Italy) 